\title{
Knowledge Collaboration Evolution Mechanisms of IT Service Outsourcing Enterprises: An Analysis Based on a Complex Network
}

\author{
Jie SUN, Guilan SHEN, Jianbin CHEN
}

\begin{abstract}
The purpose of this study is to explore the self-organization evolution mechanisms of IT service outsourcing enterprise knowledge systems. First, this paper conducts a microanalysis of knowledge activities and discusses the macro characteristics of different evolution phases of enterprise knowledge collaboration networks. Second, it proposes the dominant factors of knowledge collaboration phases. Finally, it builds a network model of enterprise knowledge systems, calculates network structure parameters to reflect the phase changes in knowledge collaboration, and analyses the evolution path of knowledge systems driven by different dominant factors. This paper uses a knowledge network model based on self-organization theory to study structural problems of enterprise knowledge system evolution, and this model provides practical and theoretical value for solving the problem of knowledge collaboration among IT service outsourcing enterprises.
\end{abstract}

Keywords: complex network analysis; evolution mechanism; IT service outsourcing; knowledge collaboration

\section{INTRODUCTION}

Knowledge collaboration (KC) has been a key research topic in the field of IT service outsourcing in recent years. With the emergence of global offshore outsourcing, IT service outsourcing enterprises are developing rapidly and are being strengthened. The transfer of tacit knowledge in the process of outsourcing enables enterprises to gain new knowledge through knowledge collaboration, thus improving service quality and capacity since the process of service outsourcing is a process of enterprise knowledge collaboration as well. However, the dependence on customer knowledge transfer and the lack of internal knowledge sharing efficiency have affected enterprise knowledge collaboration. On a micro level, knowledge collaboration emphasizes the timeliness and accuracy of knowledge transfer, and efficient knowledge collaboration accelerates the accumulation of knowledge in certain fields and partially solves the problems caused by customer dependence and tacit knowledge transfer. On a macro level, knowledge collaboration emphasizes synergistic effects and knowledge proliferation, which in turn facilitate crossorganizational and cross-project knowledge collaboration. The process of knowledge collaboration during service outsourcing contains interaction and feedback, while enterprise knowledge stock has a growing dissipative structure, which is an open and complex self-organization system. Knowledge collaboration is a problem-driven process, emphasizing situations, suitability, dynamics and knowledge proliferation. This process is clearly different from knowledge sharing and knowledge transfer. Traditional research on knowledge transfer and knowledge collaboration adopts static analysis and highlights methods of knowledge conversion and interorganizational transfer. Accordingly, this research cannot fundamentally solve the problems caused by knowledge embeddedness, staff mobility, team isolation, geographical dispersion, crossorganizational and cross-cultural nature and other features of IT service outsourcing enterprises. To solve these issues, a solution that combines people and knowledge is needed. In this context, knowledge collaboration focuses on the social network and dynamic process of the interaction between people and knowledge. Only by appropriate knowledge collaboration can people gain external knowledge and tacit knowledge and promote the innovation of knowledge.

\section{LITERATURE REVIEW}

Anklam [1] was the first to put forward that knowledge collaboration is a development trend of knowledge management. Knowledge collaboration was first defined as an organizational strategy method that can dynamically aggregate internal and external systems, business processes, technologies and relationships to maximize business performance [3]. On a micro level, knowledge collaboration is a kind of activity [16] that aims to solve problems by integrating complementary knowledge. It is also an ability of an organization [12]. It can transfer the right information to the right person at the right time [9]. On a macro level, knowledge collaboration is a knowledge activity process with the goal of knowledge innovation and the participation of multiple actors with knowledge resources [8]. Its purpose is to obtain the effect of $1+1>$ 2" [4]. It is a state and dynamic process of effective collaboration among subjects, objects and environments in knowledge management [20]. There are two dimensions of knowledge collaboration among organizations: the knowledge collaboration effect and knowledge collaboration efficiency. The knowledge collaboration effect includes knowledge capital appreciation and social capital appreciation. Knowledge collaboration efficiency reflects the accuracy and timeliness of knowledge transfer between organizations [5]. Knowledge collaboration is a process in which an enterprise and external partners build a collaborative group to combine knowledge from both internal and external sources to interact, share and integrate so that the needed knowledge can be quickly obtained and increase the heterogeneity of enterprise knowledge [6].

Therefore, the concept of knowledge collaboration emphasizes the comprehensiveness, accuracy, dynamics and knowledge proliferation of elements including knowledge, time and space, with the appropriateness of knowledge collaboration and the proliferation of knowledge outcomes being its core characteristics and providing research with macro- and micro-level perspective advantages. 
Knowledge collaboration systems are typical nonlinear complex systems that are interest-bearing and anti-entropy and evolve towards the direction away from equilibrium; thus, research on the dynamic evolution of knowledge systems is very important. Alex \& David Bennet [2] conducted some research on organizational knowledge systems from the perspectives of organizational management, organizational learning, and knowledge sharing and complex adaptive systems. While knowledge organizations lost functions, knowledge self-organization was born at the right moment [21]. Liu Qiuling and Liang Gaofei [13] analysed the formation, maintenance and evolution of the dissipative structure of an enterprise knowledge system based on the theory of dissipative structure and built an evolution path model of the dissipative structure of an enterprise knowledge system. $\mathrm{Li}$ Haibo and other scholars [14] studied the complex specialties of knowledge subjects and preliminarily established a knowledge system subject model based on complex adaptive system (CAS) theory. The kernel of complexity theory is an adaptive subject and selforganization mechanism. Yang Bo [22] analysed the process, influencing factors and causal relationship of knowledge transmission between organizations, applied a system dynamics model to carry out simulation experiments, and discussed the characteristics of activity evolution during organizational knowledge transmission. Cui Rui and Huo Mingkui [7] proposed a collaboration mechanism of industry cluster knowledge collaborative innovation, including strategy collaboration, organizational collaboration, resource collaboration and institutional collaboration. Qiu Jiangnan [18] proposed an improved collaboration evolution model of a knowledge system through social network analysis, and this model proved that in a knowledge community, individual heterogeneity has a prominent influence on the dissemination and convergence of knowledge and ideas. $\mathrm{Xu}$ Qian [17] simulated and analysed the knowledge collaboration and evolution activities of the core subjects in an innovation network and studied the influence of the knowledge collaboration and evolution activities between the core subjects on the knowledge level, knowledge growth rate, cooperation level and synergy benefit of the subjects and the whole network.

On a micro level, research on knowledge collaboration focuses on how to facilitate the establishment of knowledge collaboration mechanisms to benefit the transmission and utilization of tacit knowledge. On a macro level, this research focuses on how to measure the efficiency of knowledge collaboration to be more conducive to industrial upgrade and value chain enhancement. Not enough attention has been paid to this in previous research. In this context, this paper takes a CAS and social network analysis as theoretical guidance and the knowledge collaboration of IT service outsourcing enterprises as the research object. Through research on the correlation of all elements in a knowledge collaboration network, this paper unveils an evolution law of knowledge collaboration networks and provides a decision-making reference for improving the efficiency of enterprise knowledge collaboration from the aspects of organizational structure, enterprise culture and multiincentives.

The basic assumption of this paper is that knowledge collaboration emphasizes that through human collaboration, it realizes knowledge innovation. The main measurement indicators are the accuracy and timeliness of knowledge transmission. Tacit knowledge is highly viscous and situation-dependent. Based on the theory of social capital and knowledge capital, this knowledge solves the long-standing knowledge management dilemma caused by high-frequency mobility of employees, separation of project teams, lack of knowledge transfer motivation of contracting enterprises and other reasons. Based on the above assumption, this paper intends to study the characteristics and dominant factors in different evolution phases of knowledge collaboration, mainly including microanalysis of the knowledge activities of service outsourcing enterprises based on a twodimensional perspective of situation and process, analysis of network evolution phases and their macro characteristics of enterprise knowledge collaboration, analysis of dominant factors in enterprise knowledge collaboration evolution phases, and analysis of self-organization of the internal knowledge system of the enterprise.

\section{METHODS AND ANALYSIS \\ 3.1 Micro-Analysis of Knowledge Activities of IT Service Outsourcing Enterprises}

Due to the different strategic positions of outsourcing relationships, the resources and capacity level of IT service outsourcing enterprises vary. These enterprises can be categorized into decentralized outsourcing and benefitbased outsourcing. Decentralized outsourcing requires less of the knowledge level of contracting enterprises and is mainly reflected in knowledge collaboration for single internal areas (such as IT or IS development) with a high efficiency of collaboration but a high degree of knowledge homogenization and limited knowledge proliferation capacity. Benefit-based outsourcing requires a high degree of collaboration between contracting enterprises and receiving enterprises and mainly is reflected in knowledge collaboration of cross-organizational and cross-area elements (IT or IS development, enterprise-specific business) with a relatively low efficiency of collaboration but a high degree of knowledge homogenization and greater knowledge proliferation capacity. The difference in outsourcing relationships directly determines the difference in situations of knowledge collaboration and the relatively great difference in the elements of knowledge activities. The difference in outsourcing relationships can cause differences in relationships among knowledge subjects, knowledge subjects and other elements in different modes, and differences in situation modes of knowledge collaboration. The knowledge activities of service outsourcing enterprises are micro-processes under the control of situations. Therefore, analysis of the knowledge activities of service outsourcing enterprises should obtain the characteristics of factors of knowledge collaboration from the two-dimensional perspectives of situation and process

First, from the perspective of the situational dimension, the main factors concerning situations of knowledge collaboration are social situations, organizational situations and relationship situations. Social situations mainly consist of social, economic, political, cultural and outsourcing relationships between contracting enterprises and receiving enterprises. Organizational situations mainly examine six factors of receiving 
enterprises, including culture, strategy, organizational structure, environment, technology and operation. Relationship situations mainly focus on factors including cooperation motivation, the degree of trust and the degree of similarity in organizational situations. Szulanski [19] proposed that enterprise knowledge transfer is a process of information transmission from a source party to an accepting party happening in a certain situation. Gupta and Govindarajan [10] used task environment, structural characteristics and behaviour requirements to define the main situational variables in the knowledge flow of transnational corporations and studied the relationships between these situational variables and knowledge flow. Contextual embedding of knowledge means that enterprises must fully consider the effect of situational factors when using knowledge. The knowledge situation of an enterprise is not static but requires spending considerable time and resources to change the scope of the situation.

Second, from the perspective of the process dimension, the analysis is mainly based on the life cycle of an IT service outsourcing project. The project is a relatively complete fundamental unit of knowledge activity and is the main object of microanalysis of knowledge activity. In different stages of the life cycle, the needs for knowledge vary significantly and are mainly manifested in different degrees of tacit or explicit knowledge, different areas and different knowledge transfer priorities. Among previous research, the SECI knowledge spiral model proposed by Nonaka et al. (1995) is the most representative and explains the micro-activity of enterprise knowledge. The interaction between tacit knowledge and explicit knowledge is a spiralling process and generates four modes of knowledge transfer, including socialization (tacit knowledge transfer from individual to individual), externalization (tacit knowledge from individual to group transfers to explicit knowledge), integration (explicit knowledge transfer from group to organization) and externalization (explicit knowledge from individual to group transfers to tacit knowledge). These four modes are cycled in turn, forming a general process of innovation knowledge. Li Quanxi (2016) et al. categorized knowledge collaboration from the process perspective into knowledge sharing, knowledge transfer, knowledge obtaining, knowledge integration, knowledge application and knowledge innovation. The above research demonstrated that knowledge activities inside enterprises are not static. Knowledge collaboration happens among multiple parties on different levels; that is, there is explicit knowledge and tacit knowledge. In addition, knowledge collaboration is a process of continuous knowledge transfer, diffusion, sharing and innovation.

\subsection{Analysis of Network Evolution Phases and Macro Characteristics of Enterprise Knowledge Collaboration}

All outsourcing projects of IT service outsourcing enterprises constitute the whole knowledge system. The system is a typical open complex system that realizes selforganizational evolution during the process of information exchange with the outside. After understanding the situations and characteristics of micro knowledge activities of enterprises, this paper further analyses network evolution phases and their characteristics of knowledge collaboration.

An enterprise knowledge system must meet the conditions of a self-organization complex system. First, it should be an open complex system. The process of knowledge collaboration does not exist in isolation. It relies on knowledge transfer between enterprises and is influenced and limited by the factors of the transferor and transferee. Therefore, knowledge transfer must happen in an open and large system. Second, it is a non-balanced dynamic evolution process. By incessantly transferring and absorbing knowledge, the open system continuously breaks balance and evolves from unordered to orderly. Additionally, multiple uncertain factors play a role in the process of capacity upgrading, so it is a non-balanced evolution process. Last, it is nonlinear. The knowledge system exists and functions in a network structure, through which it communicates and diffuses according to its nonlinearity. Knowledge transfer and knowledge application are carried out in nonlinear mode.

With the evolution of the knowledge network, the enterprise knowledge system shows the characteristics of self-organization, and the state of knowledge collaboration evolution is obviously different in different stages. Each stage has dominant evolution factors and typical characteristics. From the beginning of deployment to the adaptation stage, the number of participants in the knowledge network is increasing, and the timeliness, accuracy and efficiency of knowledge collaboration are consequently constantly improving. However, the effect of knowledge collaboration increases first and then remains stable. According to research on network products, the evolution of the knowledge collaboration effect follows the path of a non-concave and non-monotonic function, as shown in Fig. 1 below.

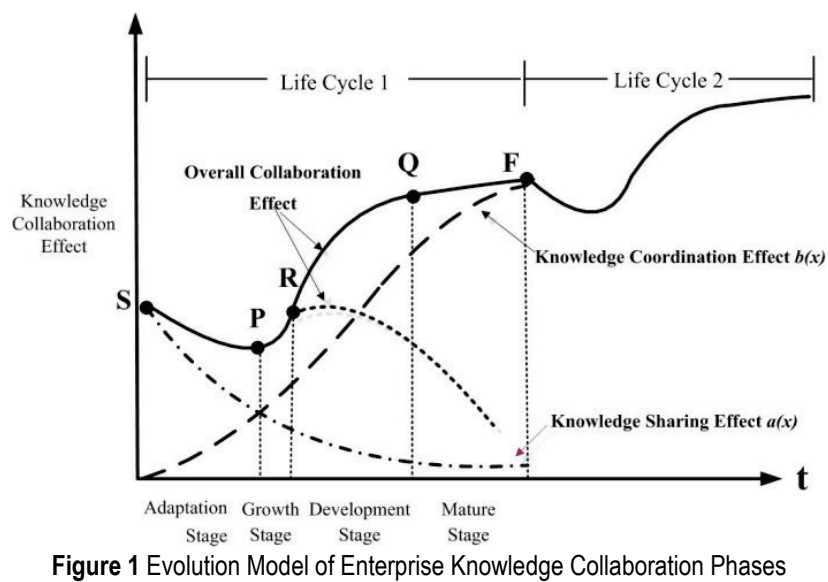

The explanation of the model is as follows: the effect of enterprise knowledge collaboration is a nonlinear and multi-life cycle. In each specific life cycle, the knowledge collaboration effect is affected by both the knowledge sharing effect $a(x)$ and the knowledge coordination effect $b(x)$. The knowledge sharing effect gradually weakens, while knowledge coordination gradually strengthens, and the overall effect of knowledge collaboration exhibits the path of increasing after dropping.

In the technology adaptation stage (S-P part in the diagram), a small number of technically sensitive employees will welcome the employment of new 
technology and actively participate in becoming a pioneer; thus, the knowledge sharing effect happens. However, before having a large scale of knowledge users, the effect of knowledge sharing will decline gradually, so the overall effect of knowledge collaboration shows a declining trend. In the growth stage (P-R part in the diagram), the scale of knowledge users expands; thus, knowledge capital and social capital accumulate gradually, and the knowledge coordination effect continues to increase, so the overall effect of knowledge collaboration begins to rise from point $\mathrm{P}$ and then exponentially increases until the development stage (R-Q part in the diagram) due to the rapid expansion of the network scale. Since the quantities of enterprise employees, customers and suppliers are limited, after the knowledge network develops to a certain scale, the effect of knowledge collaboration reaches the maximum, but the marginal effect decreases to zero when reaching the mature stage. In this stage, the knowledge system requires innovative and revolutionary knowledge elements (new source knowledge or knowledge technology) to activate the knowledge system into a new evolution cycle.

The overall effect of knowledge collaboration shown in Fig. 1 can be divided into four stages, in which four key points need to be paid special attention:

The starting point $\mathrm{S}$ of the adaptation stage: when and under what condition is the knowledge system 2.0 of the enterprise deployed?

The starting point $\mathrm{P}$ (turning point) of the growth stage: when does the effect of knowledge collaboration begin its positive growth? What are the key variables that drive the transition?

The starting point $\mathrm{R}$ (tipping point) of the development stage: how to break through the tipping point of the scale of the knowledge network? Only by breaking through this point can network evolution be truly stable. If this point cannot be broken through, then the overall effect will decrease and the life cycle will end.

The ending point $\mathrm{F}$ (saturation point): when can the point of the largest scale of the network be reached? This point means that the knowledge system is highly homogeneous, the knowledge network is highly connected, the effect of knowledge collaboration reaches the extreme value and the marginal growth is zero. To lead the knowledge system to a higher level of evolution, revolutionary knowledge factors need to be introduced to break the internal balance of the knowledge network and push the knowledge system into the next life cycle.

An analysis of the above stages is summarized in Tab. 1.

Table 1 Analysis of the Characteristics of Network Evolution Stages of Knowledge Collaboration

\begin{tabular}{|l|l|l|l|c|}
\hline \multicolumn{1}{|c|}{ Stages } & \multicolumn{1}{|c|}{$\begin{array}{c}\text { Dominant } \\
\text { Effects }\end{array}$} & KC Effect & Marginal & Key Point \\
\hline $\begin{array}{l}\text { Adaptation } \\
\text { Stage }\end{array}$ & $\begin{array}{l}\text { Knowledge } \\
\text { Sharing Effect }\end{array}$ & Decline & Decrease & $\begin{array}{c}\text { Starting } \\
\text { Point } S\end{array}$ \\
\hline Growth Stage & $\begin{array}{l}\text { Knowledge } \\
\text { Coordination } \\
\text { Effect }\end{array}$ & Increase & Increase & $\begin{array}{c}\text { Turning } \\
\text { Point } P\end{array}$ \\
\hline $\begin{array}{l}\text { Development } \\
\text { Stage }\end{array}$ & $\begin{array}{l}\text { Knowledge } \\
\text { Coordination } \\
\text { Effect }\end{array}$ & Increase & Increase & $\begin{array}{c}\text { Tipping } \\
\text { Point } R\end{array}$ \\
\hline Mature Stage & $\begin{array}{l}\text { Knowledge } \\
\text { Coordination } \\
\text { Effect }\end{array}$ & Increase & Increase & $\begin{array}{c}\text { Saturation } \\
\text { Point } F\end{array}$ \\
\hline
\end{tabular}

The above qualitative analysis of the characteristics of all stages and key points of knowledge collaboration helps to understand the characteristics and overall law of enterprise knowledge collaboration. In different evolution stages, the dominant factors are different, causing the efficiency and trajectory of different enterprise knowledge collaborations to be different.

\subsection{Analysis of the Dominant Factors of the Evolution Stages of Enterprise Knowledge Collaboration}

The above micro and macro analysis forms a factor map of spiral enterprise knowledge activity. In different evolution stages of knowledge collaboration, what are the influencing factors that dominate the transitions between stages? Existing research shows that organizational situation factors affecting knowledge transfer performance include organizational support, colleague behaviour and job requirements and that employees' motivation for knowledge transfer consists of four dimensions: emotional motivation, individual achievement motivation, interest motivation and individual interests' motivation. The above studies were carried out based on the basic structure of static balance analysis, so the existing results cannot answer questions about the dominant factors of dynamic evolution of knowledge collaboration. Therefore, this paper proposes factors for all levels that affect knowledge collaboration and discusses the dominant factors of all development stages in the process of evolution.

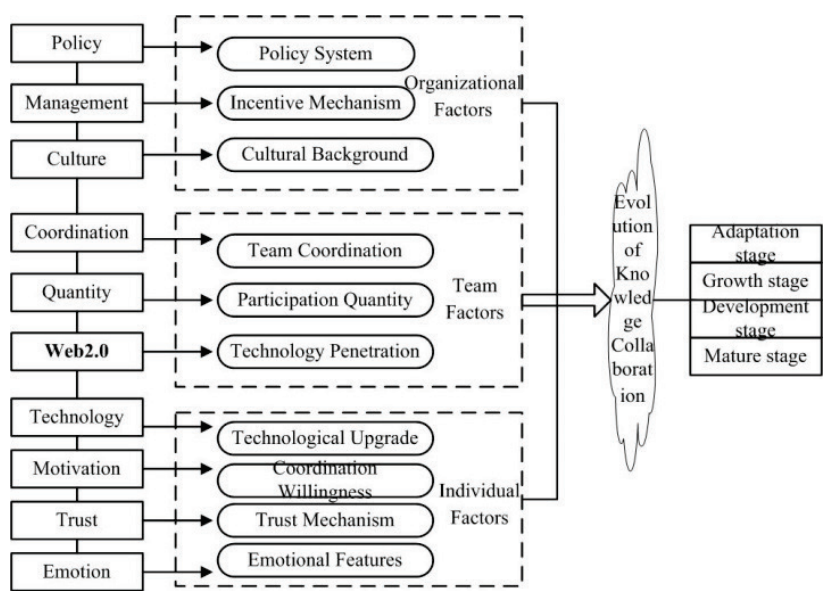

Figure 2 Dominant Factors of Evolution Stages of Knowledge Collaboration of Service Outsourcing

The dominant factors that affect the evolution of knowledge collaboration can be categorized into three levels: organizational factors, group factors and individual factors. Management strategies can be correspondingly classified into formal organization mechanisms and nonformal organization mechanisms. The former mechanisms are generally economic, short-term and measurable (such as material incentives), while the latter are social, longterm and unmeasurable (such as trust and culture).

According to the above analysis and characteristics of the knowledge subject of service outsourcing, the main influencing factors of the evolution stages of knowledge collaboration of service outsourcing enterprises are as follows in Fig. 2.

This paper supposes that the dominant factors of different evolution stages are different, and the function of 
each factor directly influences the process of evolution. In the adaptation stage of knowledge collaboration, policy systems and material incentives dominate, and policy tendencies and whether incentive mechanisms are reasonable determine the degree of technological adoption and the effect of knowledge collaboration. In the development stage, the effect of knowledge collaboration preliminarily increases, and the effect of policy incentives decreases gradually. The understanding based on coordination and mutual benefits dominates, causing rapid growth in the number of knowledge subjects and quick improvement in the knowledge collaboration effect. The accumulation of knowledge capital and social capital in turn benefits knowledge collaboration and communication and provides an inner driving force for the evolution of knowledge collaboration. In the development and mature stages, the effect of knowledge collaboration is dominated mainly by the trust and culture of the knowledge subject, and the evolution time and evolution stages are also different. In the latter stage of the mature stage, the degree of knowledge homogeneity reaches the maximum, and the growth of the effect of the knowledge network slows down and is influenced greatly by the cultural background and technological upgrading. The upgrading and replacement of technology will even directly affect knowledge collaboration in the next life cycle.

\section{DISCUSSION \\ 4.1 Orderly Degree Measurement of the Structure of an Enterprise Knowledge Network}

To study the self-organizational evolution of knowledge systems of service outsourcing enterprises, this paper builds a network model for internal knowledge of an enterprise and summarizes the law of change in the knowledge network structure, which should be reflected in the orderly degree of the knowledge network. The evolution of an enterprise knowledge system follows the path and law from disordered to ordered and then to unordered and from balance to far from balance and then back to balance. In system theory, entropy is a term used to describe and measure the disorder degree of a system, so the ordered degree of the system can be measured from dual perspectives. If measuring the overall structure from a macro perspective, structural entropy can be used to measure the degree of structural change of the system. Therefore, the structural entropy of the network can be used to measure the ordered degree of the knowledge network structure.

First, this paper describes a network with the definition of a diagram, that is, $G=(V, E)$, in which $V$ represents the collection of all nodes in the knowledge network and $E$ represents the collection of edges from nodes $V_{i}$ to nodes $V_{j}$.

The ordered degree of the network structure is:

$R=\frac{1-H}{H_{\max }}$

The larger the value of $R$ is, the higher the degree of system order.
$H$ represents the structural entropy of the knowledge system and is:

$$
H=-\sum_{i=1}^{n} p_{i} \log _{2} p_{i}
$$

In the structural entropy of the knowledge system, there are $n$ temporal states, and $p_{i}$ represents the probability of occurrence of the $i^{\text {th }}$ temporal state. $H$ represents the value of entropy, and $H_{\max }$ represents the maximum value of entropy. The size of $H$ is related to the temporal state of the system. The ratio of $H$ to $H_{\max }$ reflects the disorder state of the system, so when $H_{\max }$ reaches the maximum, the system has the highest degree of disorder.

Further studies show that the ordered degree of the network structure is also affected by the speed and quality of knowledge transmission, which reflect the efficiency and effect of knowledge transfer. Assuming that $R_{1}$ is the timeliness ordered degree of the system and $R_{2}$ is the quality ordered degree of the system, then their functions on the ordered degree of the knowledge network structure can be indicated as:

$$
R=\alpha R_{1}+\beta R_{2}
$$

where $\alpha$ and $\beta$ represent the weight coefficients that they affect.

The timeliness entropy reflects the uncertainty of the knowledge connection between nodes. The timeliness entropy of the connection between two random elements $i$ and $\mathrm{j}$ can be indicated as $H_{i}(i j)=-p_{1}(i j) \log p_{1}(i j)$. The total timeliness entropy is:

$$
H_{1}=\sum_{i}^{n} \sum_{j}^{n} H_{i j}=-\sum_{i}^{n} \sum_{j}^{n} p_{i}(i j) \log _{2} p_{1}(i j)
$$

The timeliness ordered degree of the knowledge network system is:

$R_{1}=1-\frac{H_{1}}{H_{\max }}$

The quality entropy reflects the degree of uncertainty of the quality of knowledge transfer or transmission between nodes. The timeliness entropy of the connection between two random elements $i$ and $j$ is $H_{2}(i j)=-p_{2}(i j) \log p_{2}(i j)$, so the total quality entropy is:

$H_{2}=\sum_{x=i}^{j} H_{2}(i)=-\sum_{i}^{n} p_{2}(i j) \log _{2} p_{2}(i j)=-\sum_{i}^{n} \frac{K_{i}}{A_{2}}$

The quality ordered degree of the network system is:

$R_{2}=1-\frac{H_{2}}{H_{\max }}$

The structural entropy of the knowledge network reflects the timeliness and quality of knowledge transmission in the knowledge network and can be a 
measurement of the knowledge transfer and collaboration effect. A higher degree of knowledge collaboration means improvement in the knowledge innovation efficiency and knowledge product quality, which can be used to measure the effect of knowledge transfer.

\subsection{Network Model of Self-organizational Analysis of an Enterprise Knowledge System}

Through observation of practice and qualitative analysis, it is found that the self-organization degree of a knowledge system has a nonlinear relationship with knowledge collaboration performance. The selforganization degree of the knowledge system has an optional space that corresponds to the highest value of knowledge collaboration; the self-organization process of the knowledge system is directly affected by knowledge transfer. Therefore, by adjusting the force of knowledge transfer, the evolution of the enterprise knowledge system can be influenced to maintain the corresponding optional area.

The famous philosopher Popper systematically proposed the issue of scientific knowledge growth and the prominent theory of three worlds. The mapping relationship of the three worlds is shown in Fig. 3. The objective world is objects of physics or materials; the subjective world is the state of mind about behavioural intention; and the world of knowledge is the product of human creative thinking activities that exist objectively from the subject, with autonomy and independence. There are some cognitive connections between the three worlds. The subjective world interacts with the objective world. Knowledge is the product of the subject's intellectual activities. The objective knowledge world can innovate independently, generate new knowledge, and affect the subjective world of the knowledge subject that then functions on the objective world.

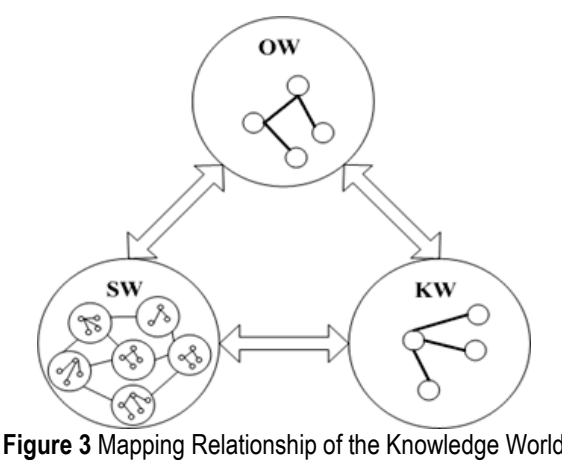

Based on the above theory, this paper believes that self-organization analysis of an internal knowledge system should consider the subject of knowledge and knowledge results, which can reflect the integration of knowledge results and the network relationship formed by the results. The knowledge network can abstract two different types of nodes according to the knowledge system: the subjective knowledge subject and objectively generated knowledge unit. The subject of knowledge mainly refers to the person who masters the knowledge. The knowledge unit mainly refers to the collection of external knowledge, including the enterprise knowledge base, process specifications, technical documents, etc. The former belongs to the subjective world, and the latter belongs to the objective world. From the perspective of the characteristics of knowledge, the subjective world belongs to the tacit knowledge of individual knowledge. The explicit expression of group knowledge determines the overall knowledge world of the enterprise organization; the $\mathrm{KW}$ is the performance of the enterprise's overall knowledge, which in turn affects the knowledge network relationship and structural evolution of the individual in the subjective world. Therefore, the self-organization evolution process of an IT service outsourcing enterprise knowledge system reflects the evolutionary development process of mutual mapping; mutual influence; and mutual creation of individual knowledge and group knowledge, tacit knowledge and explicit knowledge. Therefore, an enterprise self-organizing knowledge network system combining a "knowledge person network" and "knowledge element network" can be established.

$I$ is used to represent the internal knowledge network system of an enterprise. I includes a "knowledge person" and "knowledge element", indicated by $N_{k}(k=1,2)$, that is, $I=\left(N_{1}, N_{2}\right)$, in which $N_{1}$ represents the knowledge subject network and $N_{2}$ represents the knowledge unit network.

According to the definition of a network in graph theory, the two networks $N_{k}(k=1,2)$ in $I$ can be represented by the weighting graph $G_{k}=\left(V_{k}, E_{k}, W_{k}\right)$, where $V_{k}$ is the collection that is composed of all nodes in the two networks; $E_{k}=\left\{e_{i j}^{k}\right\}$ is the collection of all connected edges in $N_{k}$; and $W_{k}=\left\{W_{i j}^{k}\right\}$ represents the weights of all edges $e_{i j}^{k}$ in $N_{k}$, i.e., the transfer of knowledge in the network. The network structure of the internal knowledge system of an enterprise is shown in Fig. 4.

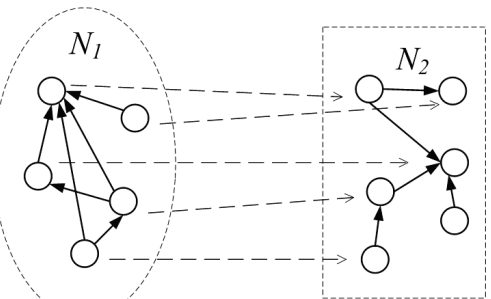

Figure 4 Network Structure of an Internal Enterprise Knowledge System

To better understand the evolution process of the network, the structural order degree of the knowledge network is used to measure the effect and upgrade state of knowledge transfer.

\subsection{Analysis of the Process of Enterprise Knowledge System Self-organization}

The self-organization evolution process of an enterprise knowledge system is driven by endogenous and conscious forward-looking behaviour, and the system acquires new knowledge through changes in the knowledge network structure. In the knowledge systems of IT service outsourcing enterprises, this self-organization evolution process represents the process of consciously acquiring and absorbing knowledge to improve their own ability and accumulate knowledge. The process promotes the formation and development of enterprise knowledge 
networks by influencing the connections between individual subjective worlds and objective knowledge worlds. If knowledge transfer is used as a regulating role of the enterprise knowledge system, it represents the influence of the dominant factors at different stages on $N_{1}$ and $N_{2}$ and acts on the self-organization evolution process of the system. The relationship between knowledge transfer and the self-organization evolution process of enterprise knowledge systems is as follows:

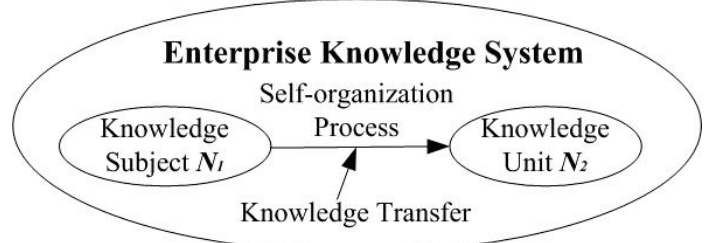

Figure 5 Relationship between Knowledge Transfer and the Enterprise Knowledge System Evolution Process

It is assumed that $M=\left(M_{i}, i=1,2, \ldots, n\right)$ represents a knowledge transfer strategy collection, including various formal and informal knowledge transfer strategies and mechanisms, which directly affect the changes in the parameters of the knowledge network and reflect the change in the network structure entropy in the process of enterprise knowledge system evolution. It is assumed that $H_{M}$ is the knowledge increment generated by the interaction between the knowledge system and the external environment, reflecting the degree of influence of knowledge transfer on the motivation and behaviour of the knowledge subject; $H_{K}$ is the knowledge increment generated by the knowledge system itself and is usually positive.

The equation for the structural entropy of the enterprise internal knowledge system can be indicated as follows:

$H=H_{M}-H_{K}$

The above equation shows that knowledge transfer will promote the generation of new knowledge entropy in the enterprise knowledge system and at the same time reduce the knowledge entropy generated by the knowledge system itself, thus ensuring improvement in the ordered degree of the whole system.

As was previously known, $R$ represents the ordered degree of the network structure, and $H$ represents the structural entropy of the knowledge network. In addition, the definition of $P$ is the effect of enterprise knowledge transfer, reflected as a function of the ordered degree of the knowledge network structure, that is, the function of knowledge structure entropy as follows:

$P=F(R)$

Where the value of $P$ can be obtained by calculating, the increase in the tangible knowledge result between the two time points and the increase in the node connectivity.

The process of change in the knowledge network structure is not a stable, balanced static convergence process. Therefore, if measured by a growing homogenization index, this self-organizing endogenous structure may exhibit a special trajectory, and a logistic diffusion equation can be used to represent this trajectory. The logistic diffusion equation is a mathematical equation describing the system's growth function. It is a common sigmoid function that exhibits exponential growth at the initial stage. As the saturation growth rate gradually slows down, it finally approaches the boundary quiescent state. The evolution process of knowledge system selforganization is an unsteady and non-balanced endogenous process. The logistic equation cannot reflect the tendency of the knowledge system structure to approach instability, but it can be used to abstractly express the state of a certain stage. This paper uses logistic equations to describe the evolution characteristics and laws of enterprise knowledge systems.

The ordered degree of the knowledge network structure $R$ will evolve over time. Assuming that the network structural entropy $H$ is a structural variable, then:

$$
H_{t}=H_{t-1}\left[1+b\left(1-\frac{H_{t-1}}{K}\right)\right]+u_{t}
$$

where $b$ is the structural coefficient, that is, the diffusion coefficient, $K$ represents the knowledge carrying capacity and $u_{t}$ is the error term. To analyse the variation in the network structural entropy, the network structuring rate can be obtained from the above equation as follows:

$$
\frac{H_{t}-H_{t-1}}{H_{t-1}}=b-b \frac{H_{t-1}}{K}+\frac{u_{t}}{H_{t-1}}
$$

Similarly,

$\ln H_{t}-\ln H_{t-1}=b-b \frac{H_{t-1}}{K}+e_{t}$

where $e_{t}=\frac{u_{t}}{H_{t-1}}$. Then, the equation is expanded to obtain:

$\ln H_{t}-\ln H_{t-1}=[b(\ldots)]\left[1-\frac{H_{t-1}}{K(\ldots)}\right]+e_{t}$

With the passage of time, the diffusion coefficient $b$ and the carrying coefficient $K$ are no longer constant and are affected by various factors, so they are represented by functions of various influencing factors. $b(. .$.$) represents a$ function of various factors affecting the diffusion coefficient, and the influencing factors in $b(\ldots)$ are generally short-term; the $K(\ldots)$ function contains various factors that affect the carrying capacity of the enterprise knowledge system. These factors determine the ultimate carrying capacity that the structural state can approach and are generally long-term effect factors.

The expanded equation can be used to analyse the state evolution process of the enterprise knowledge system to assess the stage of the system and the changes in the system structure.

A logistic diffusion curve means that the enterprise knowledge system enters the structuring stage, and the probability of unstable and discontinuous structure 
conversion increases. Therefore, two main aspects are discussed for the analysis of the evolution process of the enterprise knowledge system. First, which factors affect the structuralizing of the system? That is, what are the influencing factors of the diffusion coefficient $b$ ? Second, which factors affect the critical point of the structure? That is, what are the influencing factors of the carrying coefficient $K$ ? When the enterprise knowledge system reaches the saturation stage of a certain stage, it may transition to the next stage. At this time, the identification of the stage characteristics can help the enterprise to effectively adopt adjustment strategies or policies to help the knowledge system smoothly transition to a new state.

Based on the results of the previous discussion, these factors should depend on the knowledge base and knowledge transfer of the enterprise knowledge system. The factors affecting the system diffusivity in function $b(. .$. are short-term, generally economic and measurable, such as the flow of knowledge and the cost factor of acquiring knowledge, while the long-term factors in function $K(\ldots)$ are often non-economic and unmeasurable, such as knowledge sharing mechanisms, knowledge transfer strategies, and corporate learning culture. These two kinds of factors will also affect each other. Long-term factors often determine the time when the system structuralizing occurs. Short-term factors can lead to changes and transitions in the system state. Therefore, these factors can be regarded as "quantitative change" and "qualitative change" processes of the self-organization evolution of enterprise knowledge systems.

\section{CONCLUSIONS}

The dominant factor analysis of the evolution of knowledge collaboration in this paper follows the theoretical basis of a "social relationship". The psychological contract and relationship contract are taken as the main perspectives to carry out theoretical deduction. The characteristics of each stage of knowledge collaboration and the key points are qualitatively analysed. Based on the above analysis, this paper proposes the following main conclusions:

First, the evolution of knowledge collaboration in service outsourcing presents phased features, with each stage having different dominant factors;

Second, in the adaptation and growth stages of knowledge collaboration evolution, the formal organizational mechanism dominates; in the development and mature stages of knowledge collaboration, the informal organizational mechanism dominates;

Third, due to the self-organizing nature of the evolution of enterprise knowledge collaboration networks, formal organizational mechanisms require the intermediary role of social factors to influence the behaviour of knowledge subjects;

Fourth, with the positive evolution of the state of knowledge collaboration, the marginal benefit of overall performance shows a decreasing trend.

The next step is to further consider how to identify different stages and different governance mechanisms and provide decision-making support for how to promote knowledge collaboration in terms of factor configuration and opportunity selection.

\section{Acknowledgements}

This research was supported by Talent Selection Project 2018 of Beijing Union University (Grant No. BPHR2018DZ02), Scientific Research Project of Beijing Educational Committee (KM201711417004) and National Natural Science Foundation of China (Grant No. 71572015).

\section{REFERENCES}

[1] Anklam, P. (2001). The Camelot of collaboration. Knowledge Management Magazine, 5(2), 1-12.

[2] Bennet, A. \& Bennet, D. (2003). Designing the Knowledge Organization of the Future: The Intelligent Complex Adaptive System. Handbook on Knowledge Management, Springer Berlin Heidelberg. https://doi.org/10.1007/978-3-540-24748-7_30

[3] Karlenzig, W. (2002). Tap into the Power of Knowledge collaboration. Dimension Data, http://www.tmenet.com/.

[4] Chen, K. Y. \& Chen, K. Q. (2002). Research on enterprise knowledge collaboration. Information Science, 20(9), 986989.

[5] Chen, J. B., Guo, Y. L., \& Xu, K. B. (2014). Research on knowledge collaboration benefit evaluation based on capital value-added. Science of Science and Management of science and technology, 35(5), 35-43.

[6] Chu, J. W. \& Zhang, J. (2017). Research on the Role, Influencing Factors and Guarantee Measures of Enterprise Open Innovation Knowledge Collaboration. Modern Information, 37(1), 25-30.

[7] Cui, R. \& Huo, M. K. (2016). Construction of Knowledge Collaboration Innovation Network of Industrial Clusters. Information Science, 34(1), 155-160.

[8] Fan, Z. P., Feng, B., \& Yu, Z. C. (2007). Development and Research Prospect of Knowledge Collaboration. Science of Science and Management of science and technology, 11, 8591.

[9] Glogel, L., Howell, P,, Hugh, H. et al. (2006). Knowledge collaboration for IT support. http://www.thinkhdi.com/.

[10] Gupta, A. K. \& Govindarajan, V. (2000). Knowledge flows within multinational corporations. Strategic Management Journal, 21(4), 473-496. https://doi.org/10.1002/(SICI)1097-0266(200004)21:4<473::AIDSMJ84>3.0.C0;2-1

[11] Ikujiro, N. \& Hirotaka, T. (1995). The Knowledge-Creating Company. Oxford University Press.

[12] Leijen, H. V. \& Baets, W. J. (2002). A Cognitive Framework for Reengineering Knowledge-intensive Processes. Proceedings of the $36^{\text {th }}$ Hawaii international Conference on System Sciences (HICSS'03), IEEE.

[13] Liu, Q. L. \& Liang, G. F. (2006). Formation and Evolution of Dissipative Structure of Enterprise Knowledge System. Scientific Management Research, 24(4).

[14] Li, H. B., Liu, Z. Y., \& Ding, K. (2006). Research on Organizational Knowledge System Subject Based on Complex Adaptive System Theory. Science and Technology Management Research, (7), 199- 202.

[15] Li, Q. X., Zhang, P., \& Wang, N. (2015). Research on Knowledge Collaboration Process of Supply Chain Enterprises. Information Science, 33(7), 150-154.

[16] McKelvey, M., Alm, H., \& Riccaboni. M. (2003). Does colocation matter for formal knowledge collaboration in the Swedish biotechnology-pharmaceutical sector? Research Policy, 32(3), 483-501. https://doi.org/10.1016/S0048-7333(02)00020-3

[17] Xu Qian. (2019). Simulation Research on knowledge coevolution of emerging technology enterprises in "vertical cross" innovation network. Economic geography, (2). 
[18] Qiu, J. N., Zhang, Y., \& Xu, K. (2018). Research on Collaborative Evolution of Knowledge and Social System Based on Individual Heterogeneity in Online Knowledge Community. Operations Research and Management Science, 27(5), 119-129.

[19] Szulanski, G. (1996). Exploring internal stickiness: Impediments to the transfer of best practice within the firm. Strategic Management Journal, (17) 27-43. https://doi.org/10.1002/smj.4250171105

[20] Tong, Z. H. (2012). To explore the relationship between knowledge collaboration and relevant concepts. Library and Information Service, 56(8), 107-112.

[21] Yan, N. \& Li, Z. Z. (2001). A Preliminary Study on Knowledge Self-organization of Enterprises: A Case Study of Silicon Valley and Huawei Corporation. Information \& Knowledge, 02.

[22] Yang, B. (2010). Evolution model and Simulation of system dynamics modeling for knowledge transfer. Library and Information Service, 9(18), 89-94.

\section{Contact information:}

Jie SUN, Associate Professor

(Corresponding author)

Business College of Beijing Union University,

YanJingDongLi A3, ChaoYang District, Beijing, China, 100025

E-mail: jie.sun@buu.edu.cn

Guilan SHEH, Associate Professor

Business College of Beijing Union University,

YanJingDongLi A3, ChaoYang District, Beijing, China, 100025

E-mail: guilan.shen@buu.edu.cn

Jianbin CHEN, Professor

Business College of Beijing Union University,

YanJingDongLi A3, ChaoYang District, Beijing, China, 100025

E-mail: jianbin.chen@buu.edu.cn 